\title{
DEPFET Pixels as a Vertex Detector for the Belle II Experiment
}

\author{
Jochen Schieck for the DEPFET Collaboration \\ Ludwig-Maximilians-Universität München, Am Coulombwall 1, D-85748 Garching, Germany \\ and Excellence Cluster Universe, Boltzmannstr. 2, D-85748 Garching, Germany
}

August 14, 2019

\begin{abstract}
Currently the heavy flavour factory KEKB located at the KEK accelerator centre in Tsukuba, Japan, is being upgraded to the Super KEKB factory, aiming for a substantially higher luminosity of $8 \times 10^{35} \mathrm{~cm}^{-2} \mathrm{~s}^{-1}$. This by a factor of 40 increased luminosity requires significant modifications of the Belle detector. The vertex detector needs to be redesigned and, besides a four-layer silicon strip detector, a pixel detector based on the DEPFET technology will be installed closest to the interaction point. The requirements for this pixel detector, the DEPFET technology and the current status of the pixel detector will be presented in this paper.
\end{abstract}

\section{Introduction}

The Standard Model of particle physics (SM) is a very successful theory describing consistently all particle physics measurements being observed up to now. However, it is believed that this theory is only an effective theory since some important questions still remain open e.g. the SM does not include gravity and cannot explain the observed baryon asymmetry in the universe. The quest for physics beyond the SM is therefore the major aim of modern particle physics experiments. This search can be performed with two orthogonal approaches: production of new particles with high energy colliders (like the LHC at CERN) or through precision experiments like Belle II at the Super KEKB collider. With precision experiments new particles and new forces can appear in loop cor- rections and possible deviations from SM predictions provide a clear hint for physics effects beyond the SM. If the coupling to these new particles is large, the sensitivity to physics beyond the SM can be significantly increased compared to direct detection experiments like LHC.

\section{The Super KEKB Collider and Belle II Detector}

The Super KEKB collider is an asymmetric $\mathrm{e}^{+} \mathrm{e}^{-}$ collider $\left(\mathrm{e}^{-}: 7 \mathrm{GeV}, \mathrm{e}^{+}: 4 \mathrm{GeV}\right)$ operating at the $\Upsilon(4 \mathrm{~s})$ resonance. The operation is expected to start in 2015 and will provide $50 \mathrm{fb}^{-1}$ during 10 years operation time. Compared to the KEKB collider the boost $\gamma \beta$ is decreased leading to a deteriorate resolution for time dependent measurements. Several parts of the Belle detector have to be adapted to the new Super KEKB running conditions. A detailed description of the planned upgrade including a detailed summary of the pixel detector can be found in [1. The vertex detector of the upgraded Belle detector ("Belle II") will undergo a major revision and next to the silicon strip detector (SVD) a pixel detector (PXD) will be installed close to the interaction point. The increased luminosity will be accompanied by worse background conditions and the new pixel detector has to be able to cope with these conditions. In order to digest the high background, the detector has to provide a fast readout cycle, a high signal-to-noise ratio and the detector has to be tolerant against irradiation. To fulfil the physics re- 
quirements the detector has to have an excellent hit resolution and built with a very low material budget. A pixel detector based on the DEPFET (Depleted p-channel field effect transistor) technology [2 fulfils all these requirements.

\section{The DEPFET Pixel Detector}

\subsection{The DEPFET Principle}

A single DEPFET pixel cell consists of a field effect transistor (FET) which is operated on sideways depleted n-type bulk material. Electron-hole pairs produced by traversing particles are separated by the electric field and the electrons are collected in the internal gate, a deep n-implant below the gate of the FET. The current in the field effect transistor between the source and the drain will then be modulated by the gate of the transistor and in addition by the field generated from the electrons collected in the internal gate. After the measurement of the current the collected electrons are removed by the clear contact based on a punch-through mechanism to the internal gate. The layout of a single cell is shown in Fig. 1

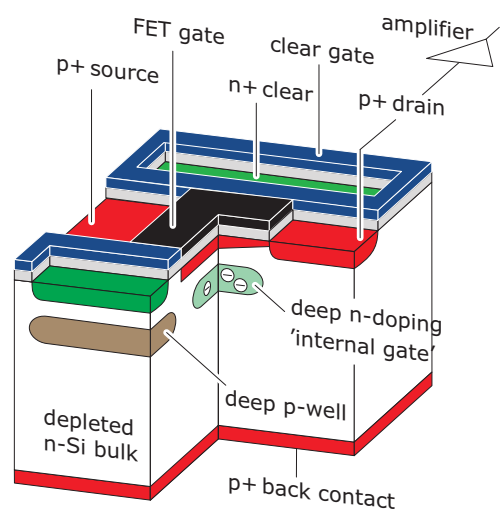

Figure 1: Sketch of a single DEPFET cell taken from [1]. Besides the FET gate the transistor current is modulated by electrons collected in the internal gate. The electrons can be removed via the clear contact.
A key parameter for the DEPFET performance is the internal amplification parameter $g_{q}$, which describes the change in transistor current $\Delta I_{D}$ divided by the charge collected in the internal gate $\Delta q_{i n}$ : $g_{q}=\Delta I_{D} / \Delta q_{i n}$. The expected number for the DEPFET sensors used in Belle II is $g_{q} \approx 400 \mathrm{pA} / \mathrm{e}^{-}$, leading to a change in the FET current of $2.4 \mu \mathrm{A}$, assuming 6000 electron hole pairs being produced in a $75 \mu \mathrm{m}$ thick silicon layer. The low power consumption is a major advantage of the DEPFET design. The detector is always sensitive to charged tracks passing the detector, while the gate is only switched on during the readout process. In the current design the active sensor area consumes only $0.5 \mathrm{~W}$ per half ladder.

\subsection{The Pixel Detector Layout}

The detector consists of two cylindrical layers, with the innermost layer located $1.4 \mathrm{~cm}$ and the outer one $2.2 \mathrm{~cm}$ away from the interaction point. The modules in the inner (outer) layer contain small pixels of size $50 \times 55 \mu \mathrm{m}^{2}\left(50 \times 60 \mu \mathrm{m}^{2}\right)$ and large pixels of size $50 \times 70 \mu \mathrm{m}^{2}\left(50 \times 85 \mu \mathrm{m}^{2}\right)$. Overall the PXD is built out of about 8 million pixel cells, arranged in 40 half-ladders, $2 \times 8$ located in the inner layer and $2 \times 12$ arranged in the outer one. Two half-ladders are front-face glued next to each other. A sketch of the Belle II pixel detector is shown in Fig. 2. The sen-

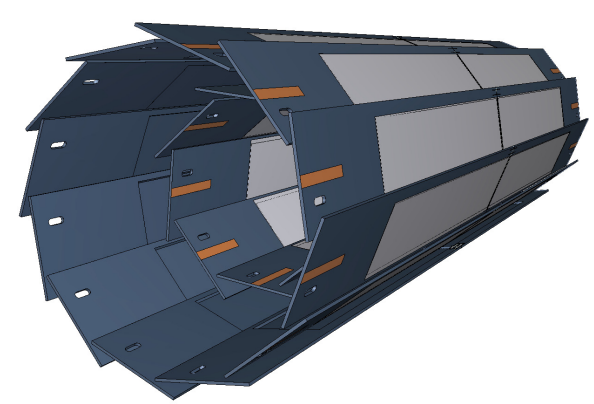

Figure 2: A sketch of the Belle II pixel detector. Each ladder consists of two half ladders and the inner layer has 8 layers while the outer one has 12 layers.

sitive area of the sensors will be thinned down to a thickness of $75 \mu \mathrm{m}$. For this the backside of the sensor 
is processed and then bonded to a so-called handling wafer. The sensor is then thinned down to the final thickness of $75 \mu \mathrm{m}$ and the required processing steps are performed on the wafer. Finally the parts of the handling wafer are etched away and only a frame will remain. This thinning process will result in an average material budget of the first layer of about $0.2 \% \mathrm{X}_{0}$, dominated by the DEPFET sensor and the frame of the module, necessary for mechanical stability. For this material budget we expect an average additional hit uncertainty of $5 \mu \mathrm{m}$ for a flight length of $1 \mathrm{~cm}$ (roughly the distance between the first and second layer) and for a track of $1 \mathrm{GeV} / c$.

The PXD will be operated in the so-called rolling shutter mode. The pixels are arranged in a grid and four pixel rows are read out simultaneously. One half ladder contains 768 rows and the processing time for a single row is about $100 \mathrm{~ns}$, dominated by the shaping time of about $70 \mathrm{~ns}$. This leads to a read out time for a complete frame of $20 \mu \mathrm{s}$, which corresponds to two complete bunch cycles. This number has to be compared to the expected event rate of about 30 $\mathrm{KHz}$, which is completely dominated by background events. With the current design we expect an occupancy of $1 \%$ in the inner layer of the PXD. A sketch illustrating the readout scheme of the PXD is shown in Fig. 3.

For steering and reading out of the PXD three types of ASICs are operated on the all-silicon halfladder. Each row of pixels is connected to a so-called Switcher, an ASIC responsible for selecting pixels to be read out by applying a voltage to the external gate and to clear the internal gate after the readout has taken place. Since four rows are selected at the same time and a single Switcher can address 32 channels at the same time six Switcher are needed for each half ladder. The current from the drain of the selected DEPFET cells is processed in the DCDB, a dedicated ASIC responsible for digitising the signal. In addition the DCDB also performs a common mode subtraction. The digitised and common mode corrected signal is then further processed by the DHP. This ASIC performs pedestal subtraction and zero suppression. For each half ladder four DCDBs and DHPs are needed for the 250 columns of DEPFET cells. The layout of a single PXD half-ladder is shown

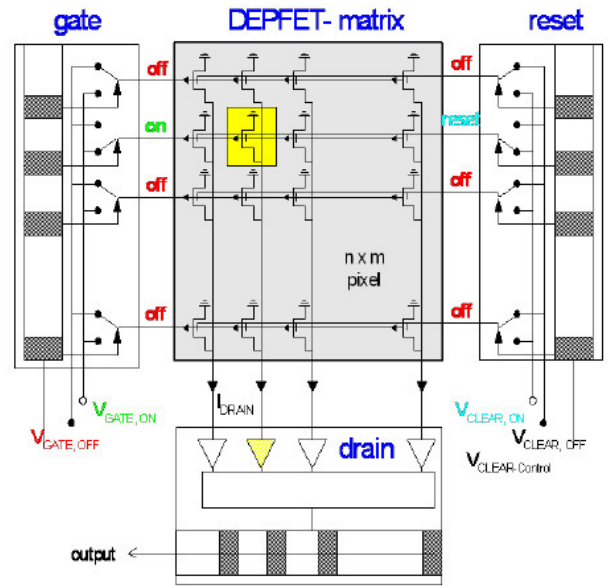

Figure 3: Sketch illustrating the rolling shutter operation of the DEPFET matrix [1].

in Fig. 4. The zero suppressed data coming from a

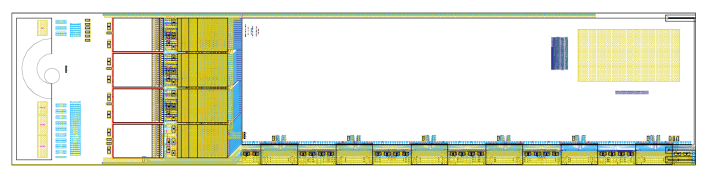

Figure 4: Layout of a PXD half-ladder. The white large area corresponds to the sensitive area, populated with $768 \times 256$ DEPFET pixels. The area below the sensitive area contains the six Switchers and left of the sensitive area the DCDB and the DHP can be found.

single half module has a data rate of about $4 \mathrm{Gbit} / \mathrm{s}$ and is transmitted via a $40 \mathrm{~cm}$ long kapton flex outside the inner detector region of Belle II. Besides the transmission of data the kapton flex is also part of the power distribution of the PXD. Each half module needs about 20 different voltages to supply the three different ASICs and the sensor. Outside the inner detector region the kapton flex is replaced by an about $15 \mathrm{~m}$ long cables to connect the PXD to the power supply modules [3] and to the data handling hybrid (DHH). The DHH is responsible for serialising the data stream and to manage the trigger and timing 
information. The data from all half-modules leads to a data rate of $58 \mathrm{Gbit} / \mathrm{s}$ [1], a size which would by far dominate the overall Belle II data rate. To further reduce the data rate originating from the PXD a further data suppression scheme is applied. Tracks online reconstructed from hits in the SVD are extrapolated to the PXD and only hits in the PXD associated to a certain region of interest defined by the SVD track are passed to the Belle II data acquisition. A significant fraction of hits originating from background events, mainly from low energy QED processes, can be rejected at an early stage.

\subsection{DEPFET Test Beam Measure- ments}

Dedicated tests of DEPFET devices are performed with test beam measurements at CERN and DESY 4]. The design of the DEPFET matrices used in these tests is not identical to the final one, but close enough in order to demonstrate the proof of principle. Full size matrices have been produced, but are not yet equipped with the ASICs. For the beam tests smaller matrices with $32 \times 64$ pixels, wire bonded to special PCBs carrying the ASICs have been used. The pixel size is different to the final design with a size of $50 \times 50 \mu \mathrm{m}^{2}$ and $50 \times 75 \mu \mathrm{m}^{2}$. The sensor thickness was thinned down to $50 \mu \mathrm{m}$. Using the ASICs for steering the matrices and reading out the DEPFET pixels the design readout time of 100 ns per pixel row was achieved. The hit efficiency achieved with this setup was more than $99 \%$. A preliminary result of the single hit resolution is shown in Fig. 5] [4]. For the DEPFET pixels with a size of $50 \times 50 \mu \mathrm{m}^{2}$ an intrinsic resolution of $12 \mu \mathrm{m}$ (RMS) was measured. The signal-to-noise ratio varies between 20 and 40, depending on the actual design of the DEPFET pixel cell.

\subsection{Radiation Hardness of the Detec- tor}

The most dangerous background events during the operation of the Super KEKB-accelerator are low momentum electron-positron pairs originating from QED processes. Two different irradiation damage

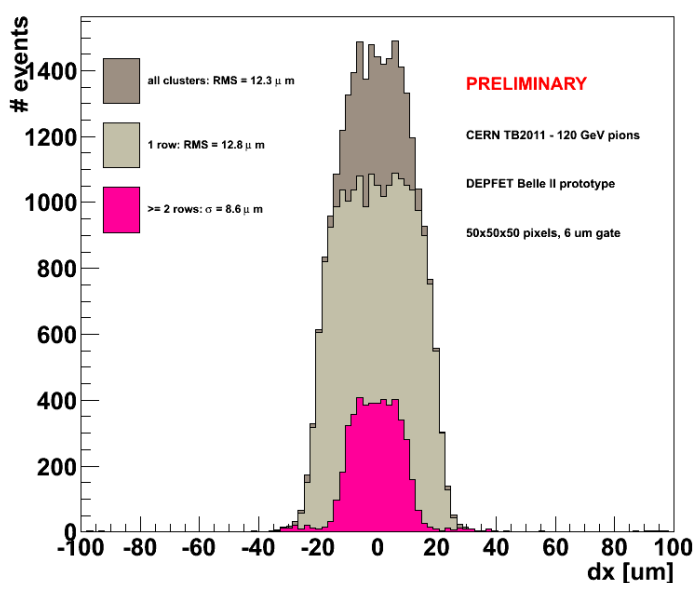

Figure 5: Residual distribution of hits collected with a DEPFET matrix during a test beam. The red distribution corresponds to hits with a signal in more than two pixel rows, while the light grey distribution corresponds to hits with a signal in a single row only. The dark grey distribution is the sum of both [4].

mechanisms can affect the operation of the PXD. Irradiation can cause damage of the silicon bulk material as well as creation of additional surface charges or oxide damage being located close to material boundaries. The two different damage types lead to different problems during the operation of the PXD. Bulk damage induces an increased leakage current with the produced charged particles ending up in the internal gate of the DEPFET. Charges created by the primary ionising particle can be trapped or detrapped, leading to changes in the signal current. Finally, in case of a very large flux, the effective doping of the device could change. However, the expected flux of $\Phi_{\text {eq }}=1.2 \times 10^{13} \mathrm{~cm}^{-2}$ for ten years of operation, assuming a hardness of electrons of 0.06 , which is based on the NIEL scaling assumption, is not expected to compromise the operation of the PXD. On the contrary surface charges and oxide damages caused by irradiation can have a significant impact on the PXD operation. Positive charges collected close to the $\mathrm{Si} / \mathrm{SiO}_{2}$ boundary lead to a shift of the gate and clear operation voltage towards more negative values. First tests using DEPFET matrices devel- 
oped for the future linear accelerator indicated a shift in the threshold voltage of more than $10 \mathrm{~V}$ during the lifetime of the PXD. A nonuniform irradiation of modules could lead to significantly different operation voltages within a single half-module. With the current design, running six switchers on a single half-module, only a limited number of different operation voltages can be applied to the individual pixels and a nonuniform irradiation can not be counterbalanced by changing the operation voltage. To reduce the sensitivity to these types of irradiation effects the outline of the DEPFET pixel cell is modified. Besides the reduction of the $\mathrm{SiO}_{2}$ layer of the DEPFET cell, leading to a reduced voltage shift, an additional $\mathrm{Si}_{3} \mathrm{~N}_{4}$ layer is incorporated which accumulates negative charges which in addition partly compensate the negative voltage shift. The flat band voltage change of a MOSFET structure similar to the one used for the DEPFET as a function of radiation dose is shown in Fig. 6 [5]. The expected dose for one year of operation is $1.85 \mathrm{MRad} / \mathrm{yr}$ and Fig. 6 indicates that the voltage shift due to irradiation is significantly reduced with no impact on the operation of the PXD.

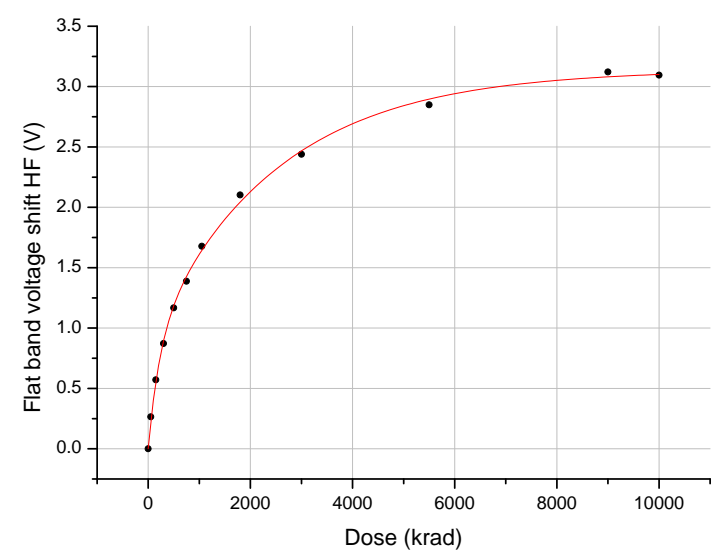

Figure 6: Flat band voltage shift of a MOSFET as a function of radiation dose. The MOSFET contains a thin $\mathrm{SiO}_{2}$ layer $(75 \mathrm{~nm})$ and an additional $\mathrm{Si}_{3} \mathrm{~N}_{4}$ layer [5].

\subsection{Thermal Management}

The low power consumption of the PXD is one of the major advantages of the DEPFET design. As already mention in section 3.1 the power consumption from the active sensor is about $0.5 \mathrm{~W}$ per half ladder. The major contribution to the total power consumption is produced by the ASICs located next to the sensitive area. During operation a power dissipation of $360 \mathrm{~W}$ is expected for the entire PXD, dominated by the readout ASICs. The DCDB and DHP are placed outside the acceptance where the ladder with the ASICs is mounted with direct thermal contact on a $\mathrm{CO}_{2}$ cooled mounting block. The switchers, being distributed along the ladder, are cooled by an air flow, with a cooling power of about $100 \mathrm{~mW} / \mathrm{cm}^{2}$. See Fig. 7 for an overview of the PXD cooling concept.

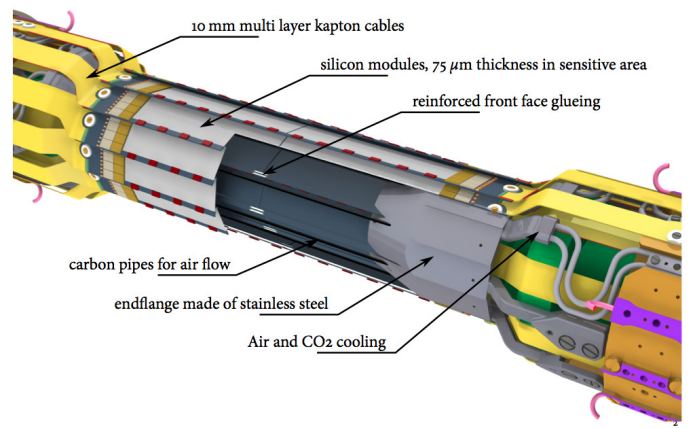

Figure 7: Overview of the cooling concept used for the PXD. The ASICs located outside the acceptance region are mounted on $\mathrm{CO}_{2}$ cooled end-flange and the switchers and the sensor is cooled by cold air.

\section{Expected Performance of the Belle II Vertex Detector}

The expected performance is estimated using simulated events 6. Charged particles reconstructed with Belle II can be described with helices and the PXD 
will mainly contribute to the impact parameter determination, $d_{0}$ and $z_{0}$, with respect of the interaction point. Fig. 8 shows the resolution of the $z_{0^{-}}$ parameter, the distance between the charged particle and the interaction point in the direction along the incoming beams, for different detector layouts. Reconstruction of charged particles in Belle II, with a vertex detector only consisting of a silicon strip detector, would lead to a similar resolution as for predecessor experiment Belle. Using in addition the information from the PXD will, as expected, significantly improve the tracking performance of the experiment. The intrinsic hit resolution of the PXD will lead to a gain in resolution for high momentum tracks, while the very low material budget will significantly improve the performance for low momentum charged particles. The study returns a similar conclusion for the resolution of the $d_{0}$-parameter.

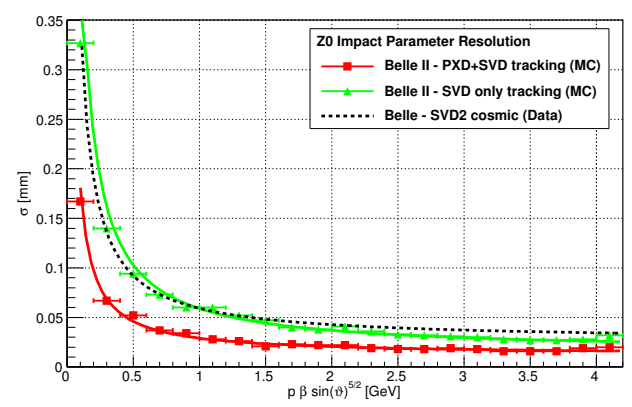

Figure 8: Resolution of the $z_{0}$-parameter of charged particles as a function of the momentum scaled with respect to the incident angle, estimated with simulated events. The green points correspond to a simulation with SVD tracking only, the red points to a simulation with SVD and PXD tracking and the dashed line is from a measurement using cosmic tracks collected with the Belle experiment [6].

\section{Summary and Conclusion}

Measurements using the Belle II-detector at the Super KEKB-accelerator offer an orthogonal approach for searches for physics beyond the SM. To exploit the full physics potential the detector is equipped with a low-material pixel detector close to the interaction point. The detector is based on the DEPFETprinciple which fulfils all requirements for a successful data taking and physics analysis at the high luminosities delivered by Super KEKB The PXD is expected to be ready for first data taking in 2015 .

\section{References}

[1] T. Abe [Belle II Collaboration], arXiv:1011.0352 [physics.ins-det].

[2] J. Kemmer and G. Lutz, Nucl. Instrum. Meth. A 253 (1987) 365.

[3] S. Rummel [DEPFET Collaboration], Nucl. Instrum. Meth. A 699 (2013) 51.

[4] The DEPFET Collaboration, Development and beam tests of DEPFET detectors for the Belle II experiment, to be published.

[5] A. Ritter, $\mathrm{PhD}$ Thesis, Radiation Hardness of DEPFET Pixel Detectors for Belle II, TU München, to be published.

[6] Z. Drasal, K. Prothmann and , PoS HQL 2012 (2012) 073 . 